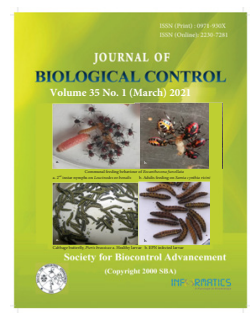

Research Article

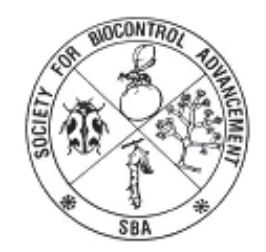

\title{
Field evaluation of biopesticides against whitefly, Bemisia tabaci (Homoptera: Aleyrodidae) in tomato
}

\author{
M. S. ABHISHEK ${ }^{1 *}$, B. C. HANUMANTHASWAMY ${ }^{1}$, T. VENKATESAN ${ }^{2}$ and K. SELVARAJ ${ }^{2}$ \\ ${ }^{1}$ Department of Agricultural Entomology, University of Agricultural and Horticultural Sciences, Shivamogga - 577204, \\ Karnataka, India \\ ${ }^{2}$ ICAR- National Bureau of Agricultural Insect Resources, Hebbal, Bellary Road Bengaluru - 560024, Karnataka, India \\ *Corresponding authorE-mail: abhigowda02@gmail.com
}

\begin{abstract}
The efficacy of different biocontrol agents, botanicals and a biorational insecticide were evaluated against whitefly, Bemisia tabaci (Hemiptera: Aleyrodidae) in tomato under field conditions at Shivamogga, Karnataka during two seasons (Rabi 2018-2019 and summer 2019-2020). The results revealed that spiromesifen 240 SC @ $0.7 \mathrm{ml} / \mathrm{L}$ was the most effective in reducing the B. tabaci population i.e., 85.12 and $85.16 \%$ reduction over untreated control during Rabi and summer, respectively followed by azadirachtin 5\%@2 ml/1 with reduction of 68.61 and $66.32 \%$ over untreated control during Rabi and summer, respectively. The response of these treatments was also observed on the yield attributes, with highest fruit yield of $53.67 \mathrm{t} / \mathrm{ha}$ (6 pickings) in spiromesifen treatment followed by azadirachtin (52.93 t/ha), but the highest Benifit: Cost ratio was noticed in azadirachtin 5\% (1:3.41), followed by spiromesifen 240 SC (1:3.38).
\end{abstract}

KEY WORDS: Azadirachtin, Bemisia tabaci, bioagents, biorational insecticide, botanicals, tomato, Spiromesifen

(Article chronicle: Received: 02-12-2020; Revised: 24-03-2021; Accepted: 27-03-2021)

\section{INTRODUCTION}

Tomato, Solanum lycopersicum L. (Solanaceae) is one of the most important vegetable crops due to its immense commercial and nutritive value and wide range of climatic adaptability. India produces about $11 \%$ of the total global tomato production and Karnataka, Andhra Pradesh, Bihar, Uttar Pradesh, Madhya Pradesh and Assam contribute major share in our country. In India, tomato is cultivated in 814 thousand ha with production of 19759 thousand tonnes during 2017-18 (Anonymous, 2018). Karnataka is the second largest producer of tomatoes and Kolar, Chikkaballapur, Bengaluru rural, Hassan, Shivamogga and Chikkamagaluru districts are major producers of tomatoes in Karnataka.

Tomato production has been intensified over the years; however, yields continue to be low like in other vegetable crops due to several production constraints such as occurrence of pests, diseases and unfavourable environmental factors. Amongst various insect pests reported in India, as many as sixteen have been observed infesting from germination to the harvesting stage which not only reduce its yield but also cause quality deterioration (Butani, 1977). The major insect pests of tomato in India are whitefly, Bemisia tabaci (Homoptera: Aleyrodidae), aphid, Aphis gossypii (Hemiptera:
Aphididae), thrips, Thrips tabaci (Thysanoptera: Thripidae), leaf miner, Liriomyza trifolii (Diptera: Agromyzidae), red spider mite, Tetranychus urticae (Acari: Tetranychidae), and fruit borer, Helicoverpa armigera (Lepidoptera: Noctuidae) (Anonymous, 2012). Among them, whitefly, B. tabaci is the major pest (David and Ananthakrishnan, 2006) and widely distributed in tropical, subtropical and temperate region. In warmer region, it is a serious pest in open field vegetable production, but crop grown under protected cultivation is equally suffering from whitefly. Both nymphs and adults suck sap from the lower leaf surface. Due to sucking the sap, yellow spots appear on the leaves followed by crinkling, curling and drying. On the other hand, this insect acts as a vector of various plant viruses and excretion of extensive honey dew attracts black sooty mould which inhibits photosynthesis thus reducing the yield. Bemisia tabaci transmit tomato yellow leaf curl virus which alone can cause 10-90\% crop loss depending upon the severity of the infestation (Palumbo et al., 2001). Bemisia tabaci is a complex of morphologically indistinguishable biotypes/genetic groups and depending on the host, cultivar, environmental conditions and the viral pathogen involved, they can cause until $95 \%$ of yield losses (Legg, 1999). Whitefly has an ability to adapt new environment and damage new hosts at different geographic regions (Basu, 1995). 
To tackle this whitefly menace, a number of chemical insecticides are liberally sprayed on tomato crop which leads to several problems like toxic residues, development of resistance, resurgence, elimination of potential natural enemies and environmental disharmony. Therefore, to reduce the above problems, bio-pesticides (natural enemies, entomopathogens and botanicals) along with recommended insecticide for the effective pest control, were attempted. These biological agents play a vital role to reduce pesticide residues, pest resistance and pest damage. Hence the present study proposed to evaluate certain bioagents and botanicals for better management of whitefly, B. tabaci in tomato.

\section{MATERIAL AND METHODS}

Field experiments were conducted at Krishi Vigyan Kendra research farm, University of Agricultural and Horticultural Sciences, Shivamogga during two seasons (Rabi 2018-19 \& 2019-2020) and (summer 2019- 2020) to evaluate the efficacy of different biopesticides, botanicals and a systemic insecticide against whitefly, Bemisia tabaci on tomato. Shivamogga belongs to the Southern Transition Zone (Zone- 7) of Karnataka with average summer temperature of 20-35 ${ }^{\circ} \mathrm{C}\left(68-95{ }^{\circ} \mathrm{F}\right)$. The majority of the rainfall occurs between June and early October.

The experiment was laid out in randomized block design with nine treatments and three replications. The treatments include entomopathogens viz., Beauveria bassiana, Metarrhizium anisopliae and Lecanicillium lecanii; predator, Chrysoperla zastrowi sillemi; biopesticides viz., azadirachtin $5 \%$, NSKE 5\% and fish oil rosin soap and biorational insecticide spiromesifen 240 SC. The entomopathogens and predator were procured from ICAR-NBAIR, Bengaluru, botanical pesticides and insecticides were obtained from private companies., The entomopathogens (Powder formulations) were tested at concentration of $5 \mathrm{gm} / \mathrm{L}$ $\left(1 \times 10^{8} \mathrm{spores} / \mathrm{g}\right)$, the predator at the rate of $6000 \mathrm{grubs} /$ ha, azadirachtin 5\%, NSKE $5 \%$ and fish oil rosin soap and spiromesifen $240 \mathrm{SC}$ at the dose of $2 \mathrm{ml} / \mathrm{L}, 5 \%, 2 \mathrm{ml} / \mathrm{L}$ and $0.7 \mathrm{ml} / \mathrm{L}$ were tested respectively.

The JK-811 hybrid seedlings were raised at KVK nursery, UAHS, Shivamogga and 25 days old seedlings were transplanted with the spacing of $90 \times 45 \mathrm{~cm}$ spacing in a plot size of $48.6 \mathrm{~m} \times 12 \mathrm{~m}$. Each subplot consists of 27 plants. All the recommended packages of practices were followed except the plant protection measures against sucking pests of tomato.

The first spray was taken up when the crop was uniformly infested with sucking pest population. Observations on the number of adult whiteflies were made on terminal three leaves of each petiole from top, middle and bottom portion of plants before and after the treatment imposition at 1, 3, 7 and 15 days. Second spray was taken up based on the rebuild up of the pest populations. The fruit yield was recorded in each treatment separately and benefit cost ratio was worked out. Further, the per cent reduction of insect population in respective treatments over the control was calculated. The per cent reduction of insect population in respective treatments over control was computed.

Per cent reduction over untreated control was also worked out using the following formula.

$$
\begin{aligned}
& \text { Per cent reduction } \\
& \text { over control }
\end{aligned}=\frac{\begin{array}{c}
\text { Pest population in control } \\
- \text { Pest population in } \\
\text { treatment }
\end{array}}{\begin{array}{c}
\text { Pest population in } \\
\text { control }
\end{array}} \text { X } 100
$$

\section{Benefit cost ratio}

Benefit cost ratio was calculated by using the following formula:

$$
\mathrm{B}: \mathrm{C} \text { Ratio }=\frac{\text { Gross return (Rs./ha) }}{\text { Total cost of production (Rs. /ha) }}
$$

\section{STATISTICAL ANALYSIS}

The population count on each replication of individual treatment was averaged to get the mean. The statistical analysis of the data was done by using Analysis of Variance (ANOVA) with Web Agri Stat Package (wasp-2) developed by ICAR, Central Costal Agriculture Research Institute, Goa. Data was transformed by square root transformation before subjecting to DMRT. The interpretation of data was done by using the critical difference value calculated at 0.05 probability level. The level of significance was expressed at 0.05 probability. After analysis, data was tabulated for interpretation of result.

\section{RESULTS AND DISCUSSION}

The results on the bioefficacy of biopesticides, botanicals and synthetic insecticide against Bemisia tabaci conducted during Rabi (2018-19 and 2019-2020) and summer (2019 and 2020) are presented here under.

\section{Bioefficacy of biopesticides against Bemisia tabaci during Rabi}

Whitefly population was observed at an early stage of tomato seedlings but treatment imposition was done at 60 days after transplanting (DAT). Treatments were not imposed up to 60 DAT, to allow the uniform build up of whitefly population in all the treatments. The data on the mean population of whiteflies of two Rabi seasons revealed that there was no 
significant difference in mean whitefly population before the imposition of treatments in all the experiment plots (Table 1). After the spray, the lowest number of whiteflies (1.10/ 3 leaves) was observed in spiromesifen $240 \mathrm{SC}$ treatment. The next best treatments which recorded the minimum number of whiteflies were azadirachtin 5\%, Lecanicillium lecanii and fish oil rosin soap with 2.32, 2.60 and 2.97/3 leaves, respectively. The maximum number of whiteflies were recorded in the treatment with predator (pesticide tolerant Chrysoperla zastrowi sillemi) (5.79/3 leaves) followed by Metarhizium anisopliae (4.82/3 leaves). The NSKE and $B$. bassiana were recorded with moderate whitefly population of 3.08 and 3.56 / 3leaves, respectively. The untreated control recorded 7.41 whiteflies/3 leaves (Table 1).

The per cent reduction of whiteflies over untreated control indicated that, the spiromesifen $240 \mathrm{SC}$ was most effective to reduce the whiteflies population over other treatments. The spiromesifen $240 \mathrm{SC}$ treated plot recorded $85.12 \%$ reduction of whiteflies over untreated control. The next best treatment with superior reduction of whiteflies was recorded in azadirachtin 5\% (68.61\%) followed by L. lecanii $(64.89 \%)$. The least reduction of whiteflies (21.85 and 34.94 $\%$ ) was recorded in C. zastrowi sillemi and M. anisopliae treatments, respectively (Table 1).

\section{Bioefficacy of biopesticides against Bemisia tabaci during summer}

The data on the mean population of whiteflies of two summer seasons revealed that there was no significant difference in mean whitefly population before the imposition of treatments in all the experiment plots. The pooled data of mean whiteflies population of two summer seasons at fifteen days after second spray varied from 1.54 to 10.35 among different treatments. The treatment spiromesifen240 SC @ $\mathrm{ml} / 1$ was recorded with lowest whiteflies population i.e., 1.54 and this was found to be superior over all other treatments. The azadirachtin 5\%@2 ml/1 was the next best treatment which recorded 3.49 whiteflies/ 3 leaves. The maximum number of whiteflies population/ 3 leaves was recorded in pesticide resistant C. zastrowi sillemi and followed by M. anisopliae where, 8.56 and 7.71 whiteflies/3 leaves, respectively (Table 2).

The per cent reduction of whiteflies over untreated control significantly varied among treatments. Maximum reduction of $85.16 \%$ was noticed in spiromesifen 240 SC treatment and was superior to other treatments. The azadirachtin 5\% treatment was next best in reducing the whiteflies population significantly over untreated control and it recorded $66.32 \%$ reduction. The lowest reduction was recorded in pesticide tolerant C. zastrowi sillemi and M. anisopliae with 17.31 and $25.53 \%$, respectively (Table 2 ).
In the present investigation, spiromesifen $240 \mathrm{SC}$ was most effective in reducing the whiteflies population 85.12 and $85.16 \%$ reduction over untreated control during Rabi and summer, respectively. These findings were in close agreement with the findings of Abhijit et al. (2018), who recorded 83.94 $\%$ reduction of whiteflies population by spiromesifen $240 \mathrm{SC}$ over untreated control. Similarly, Mahalakshmi et al.(2015) noticed $80.47 \%$ reduction of whiteflies nymphs over untreated control by spiromesifen 240 SC @ 0.4 ml/lt. and Sujay et al. (2013) recorded the lowest whiteflies population in spiromesifen240 SC @150 g a. i. ha ${ }^{-1}$ over untreated control. The significant mortality of whiteflies observed in spiromesifen treatment may be its effect on lipid biosynthesis in both adult (adulticide) and nymphs and also due to its ovicidal effect leading to longer protection. Tong (2004) reported that spiromesifen was highly toxic to nymphs and adults of $B$. tabaci but especially to young nymphs. Nauen $e t$ al., (2002) reported that application of spiromesifen markedly reduced egg hatch in whiteflies through transovariole effects upon pre-exposure of female adults. They also found that spiromesifen strongly affects fecundity of whitefly adults in a rate-dependent manner by transovariole effects.

The next best treatment was azadirachtin 5\% with whiteflies reduction of 68.61 and $66.32 \%$ over untreated control during Rabi and summer, respectively. The present findings were in line with findings of Noonari et al. (2016), who recorded $59.78 \%$ reduction of whiteflies by neem oil over untreated control in cotton ecosystem. Also Solangi et al. (2019) recorded $67.99 \%$ reduction of whiteflies over untreated control in tomato. Neem based formulations kill whiteflies in different ways (By reducing feeding, preventing larval maturity, reducing/ interrupting mating behaviour)

\section{Yield and economics (Pooled data of four seasons)}

The spiromesifen $240 \mathrm{SC}$ treated plot recorded the highest yield of $53.67 \mathrm{t} / \mathrm{ha}$ and followed by azadirachtin $5 \%$ of $52.93 \mathrm{t} / \mathrm{ha}$, fish oil rosin soap of $50.59 \mathrm{t} / \mathrm{ha}$, L. lecanii of $48.46 \mathrm{t} / \mathrm{ha}$ (Table 3). Other treatments also recorded higher yields compared untreated control (35.92 t/ha) (Table 3). The highest $\mathrm{C}: \mathrm{B}$ ratio of 1:3.41 was noticed in azadirachtin $5 \%$, followed by spiromesifen 240 SC (1:3.38), fish oil rosin soap (1:3.33) and $L$. lecanii $(1: 3.20)$. The lowest C:B ratio was noticed in pesticide resistant $C$. zastrowi sillemi (1:2.45) released plots (Table 3). Highest yield of tomato recorded in spiromesifen $240 \mathrm{SC}$ treated plot may be due to its quick knockdown effect one whitefly population and also preventing/reducing the transmission of yellow leaf curl in tomato. Alam et al. (2014) recorded significantly highest yield in spiromesifen 240 SC @ $150 \mathrm{~g}$ a.i./ha treatment followed by spiromesifen240 SC @120 g a.i./ha and also proved to be safe to the common natural enemies associated 
ABHISHEK et al.

Table 1. Bioefficacy of biopesticides against Bemisia tabaci during Rabi 2018-19 and 2019-2020 (Pooled data)

\begin{tabular}{|c|c|c|c|c|c|c|c|c|c|c|c|c|c|}
\hline \multirow{3}{*}{$\begin{array}{l}\text { Sl. } \\
\text { No. }\end{array}$} & \multirow{3}{*}{ Treatments } & \multirow{3}{*}{$\begin{array}{l}\text { Dosage } \\
\text { (gm or ml } \\
\text { per lt.) }\end{array}$} & \multicolumn{9}{|c|}{ Mean no. of whiteflies/3 leaves } & \multirow{3}{*}{$\begin{array}{l}\text { Over } \\
\text { all } \\
\text { Mean }\end{array}$} & \multirow{3}{*}{$\begin{array}{l}\text { Per } \\
\text { cent } \\
\text { ROC }\end{array}$} \\
\hline & & & \multicolumn{5}{|c|}{ First spray } & \multicolumn{4}{|c|}{ Second spray } & & \\
\hline & & & $1 \mathrm{DBS}$ & $1 \mathrm{DAS}$ & 3 DAS & 7 DAS & 15 DAS & $1 \mathrm{DAS}$ & 3 DAS & 7 DAS & 15 DAS & & \\
\hline 1. & \begin{tabular}{|l} 
Beauve- \\
ria bassiana \\
$-9\left(1 \times 10^{8}\right.$ \\
spores $/ \mathrm{ml})$
\end{tabular} & $5 \mathrm{gm}$ & $\begin{array}{c}6.09 \\
(2.56)\end{array}$ & $\begin{array}{c}5.54 \\
(2.45)^{\mathrm{abc}}\end{array}$ & $\begin{array}{c}3.95 \\
(2.10)^{\mathrm{cd}}\end{array}$ & $\begin{array}{c}2.82 \\
(1.82)^{\mathrm{c}}\end{array}$ & $\begin{array}{c}3.54 \\
(1.99)^{\mathrm{cd}}\end{array}$ & $\begin{array}{c}3.42 \\
(1.97)^{c}\end{array}$ & $\begin{array}{c}3.06 \\
(1.88)^{\mathrm{c}}\end{array}$ & $\begin{array}{c}2.73 \\
(1.79)^{\mathrm{d}}\end{array}$ & $\begin{array}{c}3.39 \\
(1.97)^{\mathrm{d}}\end{array}$ & 3.56 & 51.96 \\
\hline 2. & \begin{tabular}{|l} 
Metarhizium \\
anisopliae \\
$-41\left(1 \times 10^{8}\right.$ \\
spores $/ \mathrm{ml})$
\end{tabular} & $5 \mathrm{gm}$ & $\begin{array}{c}6.03 \\
(2.55)\end{array}$ & $\begin{array}{c}5.69 \\
(2.48)^{\mathrm{abc}}\end{array}$ & $\begin{array}{c}5.05 \\
(2.35)^{\mathrm{bc}}\end{array}$ & $\begin{array}{c}4.38 \\
(2.20)^{\mathrm{b}}\end{array}$ & $\begin{array}{c}4.98 \\
(2.33)^{\mathrm{bc}}\end{array}$ & $\begin{array}{c}4.87 \\
(2.31)^{b}\end{array}$ & $\begin{array}{c}4.55 \\
(2.24)^{\mathrm{b}}\end{array}$ & $\begin{array}{c}4.16 \\
(2.16)^{c}\end{array}$ & $\begin{array}{c}(4.86 \\
(2.31)^{\mathrm{c}}\end{array}$ & 4.82 & 34.94 \\
\hline 3. & $\begin{array}{l}\text { Lecanicil- } \\
\text { lium lecanii } \\
-32\left(1 \times 10^{8}\right. \\
\text { spores } / \mathrm{ml})\end{array}$ & $5 \mathrm{gm}$ & $\begin{array}{c}6.24 \\
(2.59)\end{array}$ & $\begin{array}{c}5.53 \\
(2.45)^{\mathrm{abc}}\end{array}$ & $\begin{array}{c}2.70 \\
(1.79)^{\mathrm{e}}\end{array}$ & $\begin{array}{c}1.44 \\
(1.39)^{\mathrm{d}}\end{array}$ & $\begin{array}{c}2.45 \\
(1.72)^{\mathrm{de}}\end{array}$ & $\begin{array}{c}2.77 \\
(1.79)^{\mathrm{cd}}\end{array}$ & $\begin{array}{c}1.95 \\
(1.56)^{\mathrm{d}}\end{array}$ & $\begin{array}{c}1.63 \\
(1.45)^{\mathrm{e}}\end{array}$ & $\begin{array}{c}2.33 \\
(1.68)^{\mathrm{e}}\end{array}$ & 2.60 & 64.89 \\
\hline 4. & $\begin{array}{l}\text { Pesticide } \\
\text { tolerant } \\
\text { Chrysoperla } \\
\text { zastrowi sil- } \\
\text { lemi }\end{array}$ & $\begin{array}{c}6000 \text { grubs/ } \\
\text { acre }\end{array}$ & $\begin{array}{c}5.87 \\
(2.52)\end{array}$ & $\begin{array}{c}5.75 \\
(2.50)^{\mathrm{abc}}\end{array}$ & $\begin{array}{c}5.60 \\
(2.47)^{\mathrm{ab}}\end{array}$ & $\begin{array}{c}5.49 \\
(2.44)^{\mathrm{ab}}\end{array}$ & $\begin{array}{c}6.23 \\
(2.59)^{\mathrm{ab}}\end{array}$ & $\begin{array}{c}5.87 \\
(2.52)^{\mathrm{b}}\end{array}$ & $\begin{array}{c}5.60 \\
(2.47)^{b}\end{array}$ & $\begin{array}{c}5.42 \\
(2.43)^{\mathrm{b}}\end{array}$ & $\begin{array}{c}6.35 \\
(2.62)^{\mathrm{b}}\end{array}$ & 5.79 & 21.85 \\
\hline 5. & $\begin{array}{l}\text { Azadirachtin } \\
5 \%\end{array}$ & $2 \mathrm{ml}$ & $\begin{array}{c}6.23 \\
(2.59)\end{array}$ & $\begin{array}{c}3.89 \\
(2.09)^{\mathrm{d}}\end{array}$ & $\begin{array}{c}2.36 \\
(1.69)^{\mathrm{e}}\end{array}$ & $\begin{array}{c}1.53 \\
(1.42)^{\mathrm{d}}\end{array}$ & $\begin{array}{c}2.73 \\
(1.76)^{\mathrm{de}}\end{array}$ & $\begin{array}{c}2.24 \\
(1.65)^{\mathrm{d}}\end{array}$ & $\begin{array}{c}1.84 \\
(1.52)^{\mathrm{e}}\end{array}$ & $\begin{array}{c}1.58 \\
(1.43)^{\mathrm{e}}\end{array}$ & $\begin{array}{c}2.44 \\
(1.71)^{\text {de }}\end{array}$ & 2.32 & 68.61 \\
\hline 6. & NSKE $5 \%$ & $5 \%$ & $\begin{array}{c}6.29 \\
(2.60)\end{array}$ & $\begin{array}{c}4.56 \\
(2.24)^{\mathrm{bcd}}\end{array}$ & $\begin{array}{c}3.11 \\
(1.89)^{\text {de }}\end{array}$ & $\begin{array}{c}2.19 \\
(1.63)^{\mathrm{cd}}\end{array}$ & $\begin{array}{c}3.39 \\
(1.95)^{\mathrm{cd}}\end{array}$ & $\begin{array}{c}3.06 \\
(1.88)^{\mathrm{cd}}\end{array}$ & $\begin{array}{c}2.80 \\
(1.81)^{\mathrm{cd}}\end{array}$ & $\begin{array}{c}2.31 \\
(1.67)^{\mathrm{de}}\end{array}$ & $\begin{array}{c}3.26 \\
(1.93)^{\text {de }}\end{array}$ & 3.08 & 58.37 \\
\hline 7. & $\begin{array}{l}\text { Fish oil rosin } \\
\text { soap }\end{array}$ & $2 \mathrm{ml}$ & $\begin{array}{c}5.92 \\
(2.53)\end{array}$ & $\begin{array}{c}4.29 \\
(2.18)^{\mathrm{cd}}\end{array}$ & $\begin{array}{c}3.09 \\
(1.89)^{\mathrm{de}}\end{array}$ & $\begin{array}{c}2.49 \\
(1.72)^{\mathrm{c}}\end{array}$ & $\begin{array}{c}3.19 \\
(1.92)^{\mathrm{d}}\end{array}$ & $\begin{array}{c}2.94 \\
(1.85)^{\mathrm{cd}}\end{array}$ & $\begin{array}{c}2.59 \\
(1.75)^{\mathrm{a}}\end{array}$ & $\begin{array}{c}2.13 \\
(1.62)^{\text {de }}\end{array}$ & $\begin{array}{c}3.03 \\
(1.87)^{\mathrm{de}}\end{array}$ & 2.97 & 59.90 \\
\hline 8. & $\begin{array}{l}\text { Spiromesifen } \\
240 \mathrm{SC}\end{array}$ & $0.7 \mathrm{ml}$ & $\begin{array}{c}6.00 \\
(2.55)\end{array}$ & $\begin{array}{c}1.90 \\
(1.53)^{\mathrm{e}}\end{array}$ & $\begin{array}{c}1.03 \\
(1.23)^{\mathrm{f}}\end{array}$ & $\begin{array}{c}0.68 \\
(1.08)^{\mathrm{e}}\end{array}$ & $\begin{array}{c}1.49 \\
(1.40)^{\mathrm{e}}\end{array}$ & $\begin{array}{c}0.97 \\
(1.21)^{\mathrm{e}}\end{array}$ & $\begin{array}{c}0.78 \\
(1.13)^{\mathrm{a}}\end{array}$ & $\begin{array}{c}0.61 \\
(1.05)^{f}\end{array}$ & $\begin{array}{c}1.36 \\
(1.36)^{\mathrm{f}}\end{array}$ & 1.10 & 85.12 \\
\hline 9. & $\begin{array}{l}\text { Untreated } \\
\text { control }\end{array}$ & - & $\begin{array}{c}6.10 \\
(2.56)\end{array}$ & $\begin{array}{l}6.19 \\
(2.58)^{\mathrm{a}}\end{array}$ & $\begin{array}{c}6.62 \\
(2.66)^{\mathrm{a}}\end{array}$ & $\begin{array}{c}6.91 \\
(2.72)^{\mathrm{a}}\end{array}$ & $\begin{array}{c}7.51 \\
(2.83)^{\mathrm{a}}\end{array}$ & $\begin{array}{c}7.56 \\
(2.84)^{\mathrm{a}}\end{array}$ & $\begin{array}{c}7.84 \\
(2.88)^{\mathrm{a}}\end{array}$ & $\begin{array}{c}8.13 \\
(2.93)^{\mathrm{a}}\end{array}$ & $\begin{array}{c}8.48 \\
(2.99)^{\mathrm{a}}\end{array}$ & 7.41 & \\
\hline & SE $m \pm$ & - & 0.09 & 0.10 & 0.10 & 0.09 & 0.13 & 0.09 & 0.08 & 0.08 & 0.09 & & \\
\hline & $\mathrm{CD}(\mathrm{P}=0.05)$ & - & 0.26 & 0.31 & 0.29 & 0.28 & 0.39 & 0.27 & 0.24 & 0.25 & 0.27 & & \\
\hline & CV $(\%)$ & - & 5.95 & 7.73 & 8.38 & 8.98 & 10.93 & 7.85 & 7.29 & 7.93 & 7.56 & & \\
\hline
\end{tabular}

NOTE: DBS: Day Before Spray; DAS: Days After Spray; Figures in the parentheses are $\sqrt{ }(x+0.5)$ transformed values; Means followed by common letters do not differ significantly by DMRT at $\mathrm{P}=0.05$ level; DMRT: Duncan's Multiple Range Test; ROC: Reduction Over Control; NSKE: Neem Seed Kernel Extract. 
Field evaluation of biopesticides against Bemisia tabaci in tomato

Table 2. Bioefficacy of biopesticides against Bemisia tabaci during summer 2019 and 2020 (Pooled data)

\begin{tabular}{|c|c|c|c|c|c|c|c|c|c|c|c|c|c|}
\hline \multirow{3}{*}{$\begin{array}{l}\text { S1. } \\
\text { No. }\end{array}$} & \multirow{3}{*}{ Treatments } & \multirow{3}{*}{$\begin{array}{l}\text { Dosage } \\
\text { (gm or ml } \\
\text { per lt.) }\end{array}$} & \multicolumn{9}{|c|}{ Mean no. of whiteflies / 3 leaves } & \multirow{3}{*}{$\begin{array}{l}\text { Over } \\
\text { all } \\
\text { Mean }\end{array}$} & \multirow{3}{*}{$\begin{array}{l}\text { Per } \\
\text { cent } \\
\text { ROC }\end{array}$} \\
\hline & & & \multicolumn{5}{|c|}{ First spray } & \multicolumn{4}{|c|}{ Second spray } & & \\
\hline & & & $1 \mathrm{DBS}$ & $1 \mathrm{DAS}$ & 3 DAS & 7DAS & $\begin{array}{l}15 \mathrm{D} \\
\mathrm{AS}\end{array}$ & 1DAS & 3DAS & 7DAS & 15DAS & & \\
\hline 1. & $\begin{array}{l}\text { Beauveria } \\
\text { bassiana }-9 \\
\left(1 \times 10^{8}\right. \\
\text { spores } / \mathrm{ml})\end{array}$ & $5 \mathrm{gm}$ & $\begin{array}{l}9.37 \\
(3.13)\end{array}$ & $\begin{array}{l}9.16 \\
(3.10)^{\mathrm{abc}}\end{array}$ & $\begin{array}{l}6.83 \\
(2.70)^{\mathrm{cd}}\end{array}$ & $\begin{array}{l}4.63 \\
(2.26)^{c}\end{array}$ & $\begin{array}{l}5.11 \\
(2.36)^{\mathrm{c}}\end{array}$ & $\begin{array}{l}4.96 \\
(2.33)^{c}\end{array}$ & $\begin{array}{l}4.76 \\
(2.28)^{\mathrm{c}}\end{array}$ & $\begin{array}{l}4.45 \\
(2.22)^{\mathrm{c}}\end{array}$ & $\begin{array}{l}4.87 \\
(2.31)^{\mathrm{c}}\end{array}$ & 5.60 & 45.94 \\
\hline 2. & $\begin{array}{l}\text { Metarhizium } \\
\text { anisopliae } \\
-41\left(1 \times 10^{8}\right. \\
\text { spores } / \mathrm{ml}) \\
\end{array}$ & $5 \mathrm{gm}$ & $\begin{array}{l}9.50 \\
(3.16)\end{array}$ & $\begin{array}{l}9.30 \\
(3.13)^{\mathrm{ab}}\end{array}$ & $\begin{array}{l}7.74 \\
(2.87)^{\mathrm{bc}}\end{array}$ & $\begin{array}{l}6.75 \\
(2.69)^{b}\end{array}$ & $\begin{array}{l}7.82 \\
(2.88)^{\mathrm{b}}\end{array}$ & $\begin{array}{l}7.69 \\
(2.86)^{b}\end{array}$ & $\begin{array}{l}7.51 \\
(2.82)^{b}\end{array}$ & $\begin{array}{l}7.24 \\
(2.78)^{b}\end{array}$ & $\begin{array}{l}7.63 \\
(2.85)^{b}\end{array}$ & 7.71 & 25.53 \\
\hline 3. & $\begin{array}{l}\text { Lecanicil- } \\
\text { lium lecanii } \\
-32\left(1 \times 10^{8}\right. \\
\text { spores } / \mathrm{ml}) \\
\end{array}$ & $5 \mathrm{gm}$ & $\begin{array}{l}9.36 \\
(3.14)\end{array}$ & $\begin{array}{l}9.13 \\
(3.10)^{\mathrm{abc}}\end{array}$ & $\begin{array}{l}5.92 \\
(2.53)^{\mathrm{de}}\end{array}$ & $\begin{array}{l}3.05 \\
(1.88)^{\mathrm{de}}\end{array}$ & $\begin{array}{l}4.14 \\
(2.15)^{\mathrm{cd}}\end{array}$ & $\begin{array}{l}3.95 \\
(2.11)^{\mathrm{cd}}\end{array}$ & $\begin{array}{l}3.65 \\
(2.03)^{\mathrm{cd}}\end{array}$ & $\begin{array}{l}3.36 \\
(1.96)^{\mathrm{cd}}\end{array}$ & $\begin{array}{l}3.89 \\
(2.09)^{\mathrm{cd}}\end{array}$ & 4.64 & 55.22 \\
\hline 4. & $\begin{array}{l}\text { Pesticide } \\
\text { tolerant } \\
\text { Chrysoperla } \\
\text { zastrowi } \\
\text { sillemi } \\
\end{array}$ & $\begin{array}{l}6000 \\
\text { grubs/ } \\
\text { acre }\end{array}$ & $\begin{array}{l}9.49 \\
(3.15)\end{array}$ & $\begin{array}{l}9.14 \\
(3.10)^{\mathrm{abc}}\end{array}$ & $\begin{array}{l}8.78 \\
(3.05)^{\mathrm{ab}}\end{array}$ & $\begin{array}{l}8.42 \\
(2.99)^{\mathrm{a}}\end{array}$ & $\begin{array}{l}8.85 \\
(3.06)^{\mathrm{ab}}\end{array}$ & $\begin{array}{l}8.62 \\
(3.02)^{\mathrm{ab}}\end{array}$ & $\begin{array}{l}8.27 \\
(2.96)^{b}\end{array}$ & $\begin{array}{l}7.92 \\
(2.90)^{\mathrm{b}}\end{array}$ & $\begin{array}{l}8.50 \\
(3.00)^{\mathrm{b}}\end{array}$ & 8.56 & 17.31 \\
\hline 5. & $\begin{array}{l}\text { Azadirachtin } \\
5 \%\end{array}$ & $2 \mathrm{ml}$ & $\begin{array}{l}9.70 \\
(3.19)\end{array}$ & $\begin{array}{l}6.50 \\
(2.64)^{d}\end{array}$ & $\begin{array}{l}3.42 \\
(1.97)^{\mathrm{f}}\end{array}$ & $\begin{array}{l}2.24 \\
(1.65)^{\mathrm{e}}\end{array}$ & $\begin{array}{l}3.58 \\
(2.01)^{\mathrm{d}}\end{array}$ & $\begin{array}{l}3.32 \\
(1.95)^{\mathrm{d}}\end{array}$ & $\begin{array}{l}2.97 \\
(1.85)^{d}\end{array}$ & $\begin{array}{l}2.56 \\
(1.74)^{\mathrm{ef}}\end{array}$ & $\begin{array}{l}3.30 \\
(1.94)^{\mathrm{d}}\end{array}$ & 3.49 & 66.32 \\
\hline 6. & NSKE $5 \%$ & $5 \%$ & $\begin{array}{l}9.30 \\
(3.13) \\
\end{array}$ & $\begin{array}{l}7.08 \\
(2.75)^{\mathrm{cd}} \\
\end{array}$ & $\begin{array}{l}5.02 \\
(2.34)^{\mathrm{e}} \\
\end{array}$ & $\begin{array}{l}3.81 \\
(2.07)^{\mathrm{cd}} \\
\end{array}$ & $\begin{array}{l}4.69 \\
(2.27)^{\mathrm{cd}} \\
\end{array}$ & $\begin{array}{l}4.34 \\
(2.19)^{\mathrm{cd}} \\
\end{array}$ & $\begin{array}{l}3.85 \\
(2.08)^{\mathrm{cd}} \\
\end{array}$ & \begin{tabular}{|l|}
3.20 \\
$(1.92)^{\mathrm{d}}$ \\
\end{tabular} & $\begin{array}{l}4.22 \\
(2.17)^{\mathrm{cd}}\end{array}$ & 4.53 & 56.28 \\
\hline 7. & $\begin{array}{l}\text { Fish oil rosin } \\
\text { soap }\end{array}$ & $2 \mathrm{ml}$ & $\begin{array}{l}9.75 \\
(3.20) \\
\end{array}$ & $\begin{array}{l}7.18 \\
(2.76)^{\mathrm{bcd}} \\
\end{array}$ & $\begin{array}{l}4.62 \\
(2.26)^{\mathrm{ef}} \\
\end{array}$ & $\begin{array}{l}3.49 \\
(1.99)^{\mathrm{cd}}\end{array}$ & \begin{tabular}{|l|}
4.27 \\
$(2.18)^{\mathrm{cd}}$ \\
\end{tabular} & $\begin{array}{l}4.00 \\
(2.12)^{\mathrm{cd}} \\
\end{array}$ & $\begin{array}{l}3.55 \\
(2.01)^{\mathrm{cd}} \\
\end{array}$ & \begin{tabular}{|l|}
3.06 \\
$(1.88)^{\mathrm{de}}$ \\
\end{tabular} & $\begin{array}{l}3.76 \\
(2.06)^{\mathrm{cd}} \\
\end{array}$ & 4.24 & 59.04 \\
\hline 8. & $\begin{array}{l}\text { Spiromesifen } \\
240 \mathrm{SC} \\
\end{array}$ & $0.7 \mathrm{ml}$ & $\begin{array}{l}9.47 \\
(3.16)\end{array}$ & $\begin{array}{l}3.70 \\
(2.04)^{\mathrm{e}}\end{array}$ & $\begin{array}{l}1.73 \\
(1.49)^{\mathrm{g}} \\
\end{array}$ & $\begin{array}{l}0.64 \\
(1.07)^{f} \\
\end{array}$ & $\begin{array}{l}1.70 \\
(1.47)^{\mathrm{e}} \\
\end{array}$ & $\begin{array}{l}1.43 \\
(1.39)^{\mathrm{e}} \\
\end{array}$ & $\begin{array}{l}1.13 \\
(1.27)^{\mathrm{e}} \\
\end{array}$ & \begin{tabular}{|l|}
0.55 \\
$(1.02)^{\mathrm{f}}$ \\
\end{tabular} & $\begin{array}{l}1.41 \\
(1.38)^{\mathrm{e}}\end{array}$ & 1.54 & 85.16 \\
\hline 9. & $\begin{array}{l}\text { Untreated } \\
\text { control }\end{array}$ & - & $\begin{array}{l}9.28 \\
(3.12) \\
\end{array}$ & $\begin{array}{l}9.46 \\
(3.15)^{\mathrm{a}} \\
\end{array}$ & $\begin{array}{l}9.65 \\
(3.18)^{\mathrm{a}} \\
\end{array}$ & $\begin{array}{l}9.95 \\
(3.22)^{\mathrm{a}} \\
\end{array}$ & $\begin{array}{l}10.38 \\
(3.29)^{\mathrm{a}} \\
\end{array}$ & $\begin{array}{l}10.51 \\
(3.31)^{\mathrm{a}} \\
\end{array}$ & $\begin{array}{l}10.72 \\
(3.34)^{\mathrm{a}} \\
\end{array}$ & \begin{tabular}{|l|l|}
10.94 \\
$(3.37)^{\mathrm{a}}$ \\
\end{tabular} & $\begin{array}{l}11.20 \\
(3.41)^{\mathrm{a}} \\
\end{array}$ & 10.35 & \\
\hline & $\mathrm{SE} \mathrm{m} \pm$ & - & 0.11 & 0.12 & 0.10 & 0.10 & 0.12 & 0.10 & 0.11 & 0.09 & 0.10 & & \\
\hline & $\mathrm{CD}(\mathrm{P}=0.05)$ & - & 0.32 & 0.37 & 0.30 & 0.29 & 0.35 & 0.30 & 0.33 & 0.28 & 0.31 & & \\
\hline & CV (\%) & - & 5.80 & 7.56 & 7.03 & 7.59 & 8.36 & 7.41 & 8.40 & 7.28 & 7.57 & & \\
\hline
\end{tabular}

NOTE: DBS: Day Before Spray; DAS: Days After Spray; Figures in the parentheses are $\sqrt{ }(x+0.5)$ transformed values; Means followed by common letters do not differ significantly by DMRT at $\mathrm{P}=0.05$ level; DMRT: Duncan's Multiple Range Test; ROC: Reduction Over Control; NSKE: Neem Seed Kernel Extract. 
Table 3. Economics of different bio agents and botanicals used against major sucking pests of tomato (pooled data of four season)

\begin{tabular}{|c|c|c|c|c|c|c|c|c|c|}
\hline $\begin{array}{l}\text { S1. } \\
\text { No. }\end{array}$ & Treatments & $\begin{array}{l}\text { Dosage gm } \\
\text { or ml per lt. }\end{array}$ & $\begin{array}{l}\text { Yield } \\
(\mathrm{t} / \mathrm{ha})\end{array}$ & $\begin{array}{l}\text { Cost of } \\
\text { cultivation } \\
\text { (Rs/ha) }\end{array}$ & $\begin{array}{l}\text { Cost of } \\
\text { protection } \\
(\mathrm{Rs} / \mathrm{ha})\end{array}$ & $\begin{array}{c}\text { Total cost of } \\
\text { production } \\
(\mathrm{Rs} / \mathrm{ha})\end{array}$ & $\begin{array}{l}\text { Gross } \\
\text { returns } \\
(\mathrm{Rs} / \mathrm{ha})\end{array}$ & $\begin{array}{l}\text { Net } \\
\text { returns } \\
(\mathrm{Rs} / \mathrm{ha})\end{array}$ & $\begin{array}{l}\mathrm{C}: \mathrm{B} \\
\text { ratio }\end{array}$ \\
\hline 1. & $\begin{array}{l}\text { Beauveria bassiana } \\
-9\left(1 \times 10^{8} \text { spores } / \mathrm{ml}\right)\end{array}$ & $5 \mathrm{gm}$ & 44.01 & 179319.98 & 2850.00 & 182169.98 & 528133.36 & 345963.38 & $1: 2.90$ \\
\hline 2. & $\begin{array}{l}\text { Metarhizium anisopliae } \\
-41\left(1 \times 10^{8} \text { spores } / \mathrm{ml}\right)\end{array}$ & $5 \mathrm{gm}$ & 38.18 & 179319.98 & 2850.00 & 182169.98 & 458135.32 & 275965.34 & $1: 2.51$ \\
\hline 3. & $\begin{array}{l}\text { Lecanicillium lecanii } \\
-32\left(1 \times 10^{8} \text { spores } / \mathrm{ml}\right)\end{array}$ & $5 \mathrm{gm}$ & 48.46 & 179319.98 & 2850.00 & 182169.98 & 581465.20 & 399295.22 & $1: 3.19$ \\
\hline 4. & $\begin{array}{l}\text { Pesticide tolerant } \\
\text { Chrysoperla zastrowi sillemi }\end{array}$ & $\begin{array}{c}6000 \\
\text { grubs/acre }\end{array}$ & 37.13 & 179319.98 & 2850.00 & 182169.98 & 445543.08 & 263373.10 & $1: 2.45$ \\
\hline 5. & Azadirachtin 5\% & $2 \mathrm{ml}$ & 52.93 & 179319.98 & 7200.00 & 186519.98 & 635167.40 & 448647.42 & $1: 3.41$ \\
\hline 6. & NSKE $5 \%$ & $5 \%$ & 44.78 & 179319.98 & 2600.00 & 181919.98 & 537392.36 & 355472.38 & $1: 2.95$ \\
\hline 7. & Fish oil rosin soap & $2 \mathrm{ml}$ & 50.59 & 179319.98 & 2920.00 & 182239.98 & 607020.04 & 424780.06 & $1: 3.33$ \\
\hline 8. & Spiromesifen $240 \mathrm{SC}$ & $0.7 \mathrm{ml}$ & 53.67 & 179319.98 & 11280.00 & 190599.98 & 644056.04 & 453456.06 & $1: 3.38$ \\
\hline 9. & Untreated control & & 35.92 & 179319.98 & 0.00 & 179319.98 & 431099.04 & 251779.06 & $1: 2.40$ \\
\hline
\end{tabular}

Market price of tomato $=$ Rs. 12,000/t; variety $-\mathrm{JK} 811$;

T1: Beauveria bassiana $-9\left(1 \times 10^{8}\right.$ spores $\left./ \mathrm{ml}\right)-\mathrm{Rs} .250 / \mathrm{kg}$

T3: Lecanicillium lecanii $-32\left(1 \times 10^{8}\right.$ spores $\left./ \mathrm{ml}\right)-\mathrm{Rs} .250 / \mathrm{kg}$

T5: Azadirachtin 5\% - Rs. 700/250 ml

T7: Fish oil rosin soap (MEENARK-L) - Rs 330/500 ml
T2: Metarhizium anisopliae $-41\left(1 \times 10^{8}\right.$ spores $\left./ \mathrm{ml}\right)-\mathrm{Rs} .250 / \mathrm{kg}$

T4: Pesticide tolerant Chrysoperla zastrowi sillemi - Rs.150/1000 grubs

T6: NSKE 5\%- Rs. 10/ 11t

T8: Spiromesifen 240 SC- Rs. 484/ $100 \mathrm{ml}$

Cost of labour (Male): Rs. 300/day; Cost of labour (Female): Rs. 250/day; Number of labours required per spray per ha =3 (1 male and 2 female); Tomato seedling cost Rs. 14,814/ha: Water volume: 500 1/ha;

with the tomato ecosystem viz., Amblyseius sp. and Stethorus sp. The reduction of natural enemies' population ranged from 2.88 to $6.48 \%$ and also Abhijith et al. (2018) reported that spiromesifen (lipid bio-synthesis inhibitor) alone showed good potency against whiteflies with no toxicity towards the non-target organisms i.e., natural enemies. The reduced effect of spiromesifen on other natural enemies like, coccinellids, spiders and lacewings might helped to reduce other insect pests damage and leading to increased yield of tomato. However, the highest benefit-cost ratio of 1:3.41 was recorded in azadirachtin 5\% treated plot because of its low price compared to cost of spiromesifen 240 SC. The present findings are similar to that of Asmita and Ukey (2014) findings.

\section{ACKNOWLEDGEMENT}

The authors are grateful to Dr. B. Ramanujam, Principal Scientist (Plant Pathology) ICAR-NBAIR, Bengaluru-560 024, Karnataka, India for providing bioagents culture for field evaluation.

\section{REFERENCES}

Abhijit G, Chatterjee ML and Anjan B. 2018. Field bioefficacy of some new insecticides and tank mixtures against whitefly on cotton in new alluvial zone of West Bengal. Pestic Res J. 30(1):1-7.
Alam SKF, Patra B, Moulita C, Dey PK, Dhar PP, Samanta $A$ and Somchoudhury AK. 2014. Bio efficacy of spiromesifen $240 \mathrm{Sc}(\mathrm{w} / \mathrm{v})$ against whitefly and red spider mites infesting tomato. J Entomol Res., 38(2): 01-10.

Anonymous. 2015. NICRA team of Tomato Pest Surveillance 2012. Manual for Tomato Pest Surveillance. Jointly published by National Centre for Integrated Pest Management (NCIPM), New Delhi, Central Institute for Dryland Agriculture, Hyderabad, Indian Institute of Horticultural Research, Bengaluru and Indian Institute of Vegetable Research, Varanasi. 39p.

Anonymous. 2018. Horticultural statistics at a glance.

Asmita S and Ukey S P. 2014. Evaluation of certain botanicals, microbials: An conventional insecticide against mealybug infesting tomato. Int J Curr Microbiol Appl Sci. 3(11): 385-389.

Basu AN. 1995. Bemisia tabaci (Gennadius): Crop pest and principal whitefly vector of plant viruses. Westview Press, New Delhi, 183p.

Butani DK. 1977. Insect pest of vegetables-tomato. Pesticides, 11: 33-36. 
David BV, Ananthakrishnan TN. 2006. General and Applied Entomology. Second Edition. Tata McGraw-Hill Publishing Company Ltd. 27p.

Legg JP. 1999. Emergence spread and strategies for controlling the pandemic of cassava mosaic virus disease in East and Central Africa. Crop Prot. 18: 627-637.

Mahalakshmi MS, Sreekanth M, Adinarayana M and Rao YK. 2015. Efficacy of some novel insecticide molecules against incidence of whiteflies (Bemisia tabaci Genn.) and occurrence of Yellow Mosaic Virus (YMV) disease in urdbean. Int J Pure Appl Biosci. 3(5): 101-106.

Nauen R, Bretschneider T, Bruck E, Elbert A, Reckmann U, Wachendorff U and Tiemann R. 2002. BSN 2060: A novel compound for whitefly and spider mite control. The BCPC Conference: Pests and Diseases, Vol. 1 and 2. Proceedings of the International Conference, Brighton, UK, 18-21 November, 2002. British Crop Protection Council, Farnham, UK, pp. 39-44.
Noonari AM, Abro GH, Khuhro RD and Buriro AS. 2016. Efficacy of biopesticide for management of sucking insect pest of cotton, Gossypium hirsutum (L.). J Basic Appl Sci. 12: 306-313.

Solangi BK, Suthar V, Riffat S, Abdul RAT, Muhammad $\mathrm{N}$ and Solangi MN. 2019. Screening of bio pesticides against insect pests of Tomato. Eur Acad Res. 5(2): 700718 .

Sujay A, Anand B, Shankarganesh S and Karuppan. 2013. Efficacy of newer insecticides against leaf hopper and whitefly infesting brinjal and its effect on coccinellids. Indian Jf Plant Prot 25: 6-11.

Tong XL. 2004. Toxicity and efficacy of spiromesifen, a tetronic acid insecticide, against sweet potato whitefly (Homoptera: Aleyrodidae) on melons and collards. Crop Prot. 23: 505-513. 